\title{
La Historia de la Universidad en la Fiesta de la Ciencia en España
}

\author{
The History of the University \\ at the Science Festival in Spain
}

\author{
José Maria Hernández Díaz \\ Universidad de Salamanca \\ ORCID ID: 0000-0001-7604-1544
}

Recibido: $\quad 21 / 04 / 2020$

Aceptado: $\quad 24 / 09 / 2020$

DOI: https://doi.org/10.20318/cian.2020.5794

Resumen: Todas las universidades de España celebran cada año académico, en el inicio de curso, un conjunto de actos que forman parte de lo que podemos denominar la Fiesta de la Ciencia, utilizando un lenguaje positivista del siglo XIX. Dentro de esa celebración alcanza especial significado la lección inaugural, que pronuncia un profesor elegido al efecto, y que suele ser un académico de prestigio en su especialidad académica. De manera paciente hemos formado primero una base de datos de 2165 unidades, discursos o lecciones inaugurales pronunciadas en las 87 universidades españolas, públicas y privadas, desde 1939 a 2019. Más tarde hemos podido formar varias categorías de análisis, que acogen diferentes campos temá-
Abstract: Every academic year, at the beginning of the course, all the universities in Spain hold a series of events that are part of what we can call the Science Festival, using a 19 th century positivist language. Within this celebration, the inaugural lesson, which is delivered by a teacher chosen for this purpose, and who is usually an academic of prestige in his academic specialty, acquires special significance. In a patient manner we have first formed a database of 2165 units, speeches or opening lessons delivered at the 87 Spanish universities, public and private, from 1939 to 2019. Later we have been able to form several categories of analysis, which host different thematic fields of university life. One of these categories is the "History of Univer-

*jmhd@usal.es 
ticos de la vida universitaria. Una de esas categorías es la "Historia de las Universidades", que nos permite obtener un cupo de 54 unidades o lecciones inaugurales que abordan esa temática. De su lectura y estudio obtenemos conclusiones muy significativas para la historia particular de algunas universidades, en especial para las consideradas como clásicas, pero también para el conjunto de la universidad española, desde sus orígenes en la Edad Media al siglo XXI. Se muestra así cómo la historia de las universidades, a través de una fuente documental propia como son las lecciones inaugurales, puede afianzarse como un campo propio dentro de los estudios sobre educación superior, y también sobre la historia institucional

Palabras clave: Historia de las Universidades, Fiesta de la Ciencia, lecciones inaugurales, siglos XX y XXI. sities", which allows us to obtain a quota of 54 units or inaugural lessons dealing with this topic. From his reading and study we obtain very significant conclusions for the particular history of some universities, especially those considered as classics, but also for the whole of the Spanish university, from its origins in the Middle Ages to the 21st century. It is shown as well as the history of the universities, through a documentary source such as the opening lessons, can be consolidated as a field within the studies on higher education, and also on the institutional history.

Keywords: History of Universities, Science Festival, Opening Lessons, 20th and 21st Centuries.

\section{Introducción}

La universidad es una de las instituciones sociales de más larga trayectoria histórica, para el caso de España hoy formada por casi un centenar de unidades, que a su vez en la actualidad acoge a un millón largo de componentes, entre alumnos, profesores, personal de apoyo, y recibe complementos materiales y sociales de un número indeterminado de instituciones e individuos de la sociedad. Este conjunto de unidades de apoyo y de referencia hoy hacen de la universidad una de las instituciones más polifacéticas, socialmente ricas, imprescindibles para la ciencia y la sociedad.

También, como es propio de toda institución, deja su rastro en la historia mediante las huellas de alguna de las muchas circunstancias que la definen o componen. Una de ellas es su organización, la distribución del espacio y del tiempo, la organización de su calendario docente, por ejemplo, porque desde su origen la principal misión que ha de atender es la formación de profesionales expertos entre las nuevas generaciones de jóvenes en un periodo de tiempo concreto y limitado.

En la organización de sus tiempos y calendarios desde hace siglos que las universidades marcan algunos días como emblemáticos para dar inicio a sus actividades. Convierten en especial el día de la inauguración del curso académico (durante siglos fue San Lucas en el mes de octubre el día del pistoletazo de salida de las actividades académicas), con celebración alegre, 
boato, procesión académica, música y ropajes. En ese mismo acto también se pronuncia una lección o conferencia inaugural, emulando los ritos eclesiásticos medievales. En esa lección inaugural, de alto significado simbólico, social y académico, por parte del orador elegido se trasladan a los oyentes reflexiones sustantivas sobre algún aspecto de la ciencia, que también quedan recogidas en documento escrito e impreso para conocimiento general. A esos actos y lección inaugural se les comienza a atribuir el nombre de Fiesta de la Ciencia en el siglo XIX por influencias positivistas.

En ese marco de referencia nos parecía oportuno revisar cómo y por qué en muchas de esas lecciones inaugurales por parte del académico de turno elegido se abordan cuestiones específicas de la historia de la universidad, de la propia o de las del conjunto de España, por un ejercicio de curiosidad científica, y por el valor añadido que representa la historia de la universidad en la comprensión explicativa del presente universitario, de posible interés para estudiantes y especialistas en este campo de estudio.

Mediante una revisión pausada de 2165 unidades de análisis, en este caso lecciones inaugurales pronunciadas en universidades españolas entre 1939 y 2019, podemos seleccionar un elenco adecuado de las que se perfilan hacia la historia de las universidades de manera directa, más allá de otras que aquí no consideramos pero que abordan asuntos varios de las diferentes ciencias y saberes investigados y enseñados. Esas lecciones nos enriquecen históricamente, tanto a la universidad de procedencia como a quienes en España cultivamos el campo especifico de la historia de las universidades.

\section{La Fiesta de la Ciencia}

La universidad en España es una institución viva de nuestro siglo XXI, pero con arraigadas resonancias medievales y renacentistas, y en consecuencia eclesiásticas, en algunas de sus expresiones externas y formas de organización y visibilidad pública. La ceremonia, el protocolo, la música, el espaciado de tiempos, la vestimenta y el discurso forman parte de la estrategia del poder cultural y de la construcción de identidades. Eso también ocurre en nuestra universidad española contemporánea.

La universidad es heredera también de pautas de comunicación oficial y emblemática, que en su día representó la lectio medieval, como modelo y espacio didáctico confirmatorio de la excelencia académica de un saber o de una ciencia, y desde luego de un catedrático. Al paso de los siglos se ha mantenido esa tradición, entre eclesiástica y académica, de dar inicio a las activi- 
dades lectivas de un curso académico con una celebración, en parte religiosa y en parte científica, pero siempre adornada con las expresiones de poder que siempre destila el protocolo que manejan los poderosos, y la universidad socialmente lo es desde su origen.

Con la reforma universitaria de los liberales a partir de 1845 (Plan Pidal) poco después se va a generalizar con carácter obligatorio que todas las universidades han de tener previsto que en el inicio de cada curso se pronuncie un discurso inaugural por parte de alguno de sus catedráticos, y casi siempre de forma rotatoria y excluyente, para una vez en la vida. De ahí la diversidad temática de las lecciones y de los oradores postulados. En un momento determinado, ciertos sectores universitarios en España, de procedencia positivista y defensores de una mayor secularización en la vida interna de las universidades, reclaman que ese día inaugural pase a denominarse Fiesta de la Ciencia ${ }^{1}$. De esa manera comenzaban a despojarse un poco los actos de celebración del alumbramiento de curso académico del ropaje eclesiástico tradicional antes comentado.

Al ser la Fiesta de la Ciencia un día tan señalado, que se celebra en un lugar señalado tan especial como es el paraninfo, y que tiene como protagonista principal al catedrático elegido para pronunciar su lección, y siendo un acto al que están invitados componentes académicos, pero también personalidades de la vida social, política, eclesiástica, empresarial y pública del entorno, la Fiesta de la Ciencia alcanza un significado simbólico extraordinario en la construcción de la identidad de la institución ante las autoridades,los asistentes, y ante quienes más tarde leen el texto impreso de la lección académica o los reportajes periodísticos del acto en la prensa y otros medios de comunicación.

\section{La Historia de la Universidad en la Fiesta de la ciencia. Antecedentes}

La historia de las universidades es un ámbito de estudio con personalidad propia y rica tradición científica que ha encontrado en España cultivadores de muy diferente procedencia, pero en especial desde la Historia del Derecho, la Historia Moderna y la Historia de la Educación, y de forma más colateral desde la Historia Contemporánea. Congresos especializados en historia

\footnotetext{
${ }^{1}$ Romero Díaz, Pedro. Conquistas que el hombre ha hecho en los dominios de la ciencia y beneficios que aquél ha recibido de ésta. Discurso pronunciado en la Universidad de Salamanca en la inauguración del curso 1862-1863. Salamanca: Impr. de Diego Vázquez, 1862, p. 5.
} 
de las universidades y números monográficos de revistas científicas sobre el tema dan fe de ello.

Sin remontarnos a obras clásicas anteriores a los últimos cincuenta años, baste mencionar la inmensa obra de Mariano Peset desde la Universidad de Valencia y su hermano José Luis desde el CSIC, el trabajo realizado desde el Centro de Historia de la Universidad de Salamanca Alfonso IX dirigido por Luis Enrique San Pedro, el Centro Antonio de Nebrija de la Universidad Carlos III de Madrid, la revista CIAN y varios monográficos de Historia de la Educación. Revista Interuniversitaria $(1984,1986,2015)$ y otros muchos artículos en ella recogidos, sobre el tema que nos ocupa, "Historia de las Universidades". Además, con independencia de obras y grupos internacionales, como el de la Universidad de Bolonia, cada universidad española de las de larga tradición cuenta con su respectiva historia, o más bien en plural en el caso de alguna de ellas. No vale la pena mencionar aquí ninguna, pero las hay de altísimo nivel, y alguna con varios volúmenes como respaldo.

En muchos de los miles de estudios existentes sobre la historia de las universidades españolas, en particular ahora sobre su etapa contemporánea, se ha utilizado el discurso inaugural del curso académico universitario como pieza documental con personalidad, y con información cualificada a veces, para apoyar otras fuentes de información documental ${ }^{2}$. Ello se justifica por la calidad científica e informativa de muchos de esos discursos, pronunciados por catedráticos seniors y reconocidos en su especialidad.

Además, la lección inaugural ha comenzado a ser estudiada como un capítulo propio de la historia de las universidades, e incluso dentro de ellas se ha realizado algún apunte sobre la historia de la universidad en la lección inaugural.

Así sucede con varios de los discursos pronunciados en las universidades españolas entre 1845 y $1936^{3}$ (Hernández Díaz, 2015; 2012). Por ejemplo, en el primer tercio del siglo XX se pronuncian varios de ellos destinados a legitimar o ensalzar la historia de alguna de las universidades, $\mathrm{o}$

${ }^{2}$ Rubio y Borrás, Manuel. Aperturas de curso en la Universidad de Barcelona desde 1536 a 1919. Reseña histórica y bibliográfica. Barcelona: Editorial Cultura, 1919; Domínguez Cabrejas, Rosa. "El discurso pedagógico a través de los paraninfos. Universidad de Zaragoza (19001923)", en X Coloquio de Historia de la Educacion. La Universidad en el siglo XX. España e Iberoamérica. Murcia: Universidad de Murcia, 1998.

${ }^{3}$ Hernández Díaz, José María. "El discurso pedagógico en la Fiesta de la Ciencia en la universidad deseada: España 1900-1936". Historia de la Educación. Revista Interuniversitaria. 34, 2015, 103-138.; Hernández Díaz, José María. “La pedagogía en la Fiesta de la Ciencia de la Universidad de Salamanca (1845-1874)”. REDEX. Revista de Educación de Extremadura. 4,2012, 49-69. 
aspectos concretos de las mismas. Así, nos encontramos con los discursos pronunciados en las universidades de Madrid en 1914 por Bonilla San Martín ${ }^{4}$, en Santiago de Compostela en 1917 por Cabeza de León ${ }^{5}$, en Salamanca en 1912 por Urbano González de la Calle ${ }^{6}$, en Sevilla en 1907 y 1922 por Joaquín Hazañas ${ }^{7}$, en Murcia en 1921 por Loustau ${ }^{8}$, en Salamanca en 1933 por Maldonado de Guevara ${ }^{9}$, en Granada en 1928 por Martos de la Fuente ${ }^{10}$, en Valencia en 1911 por Riba García ${ }^{11}$, en La Laguna en 1931 por Serra Rafols ${ }^{12}$, en Salamanca en 1925 por Téllez de Meneses ${ }^{13}$ o en Barcelona en 1926 por Torre del Cerro $^{14}$. La mayor parte de los capítulos universitarios estudiados

${ }^{4}$ Bonilla San Martín, Adolfo. La vida corporativa de los estudiantes españoles en sus relaciones con la historia de las universidades. Discurso leído en la Universidad de Madrid en la inauguración del curso 1914 a 1915. Madrid: Impr. Colonial, 1914, p. 144.

${ }^{5}$ Cabeza de León, Salvador. La disciplina en la Universidad de Santiago en el siglo XVI-XVIII. Discurso inaugural del curso 1917 a 1918 en la Universidad de Santiago. Santiago de Compostela: Impr. Paredes, 1917, pp. 26.

${ }^{6}$ González de la Calle, Urbano. Estudio de la vida académica y profesional del Brocense en nuestra Escuela. Discurso pronunciado en la Universidad de Salamanca en la inauguración del curso 1912 a 1913. Salamanca, Tip., Núñez, 1912, pp. 192

${ }^{7}$ Hazañas y la Rúa, Joaquín. La vida escolar universitaria en Sevilla (XVI-XVIII). Discurso pronunciado en la Universidad de Sevilla en la inauguración del curso 1907 a 1908. Sevilla: Papelería Sevillana, 1907, pp. 20; Hazañas y la Rúa, Joaquín. La Universidad de Sevilla. Discurso pronunciado en la Universidad de Sevilla en la inauguración del curso 1922 a 1923. Sevilla, Tip. Eulogio de las Heras, 1922, pp. 33.

${ }^{8}$ Loustau Gómez de Membrillera, José. La Universidad de Murcia y el organismo social. Discurso leído en la inauguración del curso de 1921 a 1922 en la Universidad Literaria de Murcia. Murcia: Imp. José A. Giménez, 1921 pp. 86.

${ }^{9}$ Maldonado de Guevara, Francisco. Salamanca y su universidad. Discurso pronunciado en la Universidad de Salamanca en la apertura del curso de 1933 a 1934. Salamanca: Impr. Núñez, 1933, pp. 27.

${ }^{10}$ Martos de la Fuente, José. La universidad española hasta el siglo XVIII y sus rasgos característicos. Discurso leído en la Universidad de Granada en la apertura del curso 1928 a 1929. Granada: Tip. López de Guevara, 1928, pp. 44.

${ }^{11}$ Riba García, Carlos. La universidad valentina en los años de la Guerra de la Independencia (1807-1815). Discurso leído en la solemne apertura de los estudios del año académico de 1910 a 1911 de la Universidad de Valencia. Valencia: Universidad de Valencia, 1911, pp. 147.

${ }^{12}$ Serra Rafols, Elías. Una universidad medieval, el Estudio General de Lérida. Discurso pronunciado en la Universidad de La Laguna en la apertura del curso 1931 a 1932. Madrid: Victoriano Suárez, 1931, pp. 94

${ }^{13}$ Téllez de Meneses Sánchez, José. La Universidad Salmantina y su riqueza bibliográfica. Discurso pronunciado en la Universidad de Salamanca en la apertura del curso académico de 1925 a 1926. Salamanca: Imp. Núñez, 1925.

${ }_{14}$ Torre del Cerro, Antonio de la. Provisión de cátedras en la Universidad de Barcelona de 1559 a 1596. Discurso pronunciado en la Universidad de Barcelona en la inauguración del curso 1926 a 1927. Barcelona: Núñez y Cos, 1926, pp. 26. 
en estos discursos inagurales se centran en las universidades clásicas, su organización, sistemas de selección de cátedras, estilos de vida universitaria, conductas de los estudiantes, reformas de la universidad en el periodo de la Ilustración, o personalidades muy brillantes de profesores en algunas de ellas , como Luis Vives o el Brocense.

Desde una valoración de conjunto, estos discursos adoptan una posición instrumental ante la historia de la universidad, como es su concepción legitimadora de grandezas o de reproches, del peso de los catedráticos o de los estudiantes, y en alguno de ellos se percibe un decidido sesgo involucionista frente a las reformas universitarias que comienzan a aplicarse con la creación de la Junta de Ampliación de Estudios en 1907, caso de Bonilla San Martín.

\section{Metodología y fuentes utilizadas}

Nos pareció de interés estudiar el peso que la historia de la universidad ha merecido en las lecciones inaugurales que se pronuncian año tras año en el conjunto de la universidad española en los últimos ochenta años (19392019), una vez superado el corte de la guerra civil.

Ya no hablamos solamente de 12 universidades clásicas, más las dos confesionales de la Compañía de Jesús (Deusto y Comillas), existentes hasta ese corte del conflicto bélico, sino de un volumen muy superior de ellas, que alcanza hasta las 87 universidades existentes en España en la actualidad, de las que 50 son públicas y 37 privadas (de ellas confesionales -16- o mercantiles -21-).

De manera paciente y metódica, a través de consultas a redes, catálogos, bibliotecas, repositorios documentales de diferente signo, hemos construído una base documental de 2.165 lecciones inaugurales pronunciadas anualmente en las universidades españolas en el início de cada curso académico, y publicadas (o algunas difundidas por youtube), que corresponden a la suma de la inmensa mayoría de casi todas las universidades, pues alguna muy en particular no lo ha editado. Es evidente que cada año transcurrido es preciso alimentar esa base de datos con al menos 80 nuevas lecciones inaugurales.

Sobre ese período de tiempo analizado, de 80 años, que ha producido una masa informativa imponente sobre la vida de las universidades españolas (2.165 unidades de análisis), hemos aplicado una distribución por categorías dominantes, entre las que hemos asignado un espacio a "la historia de la universidad", y ahí hemos situado aquellos discursos que tienen una orientación decidida y expresa en el tema de la historia universitaria. 
El resultado final ha sido de 54 lecciones inaugurales seleccionadas, que son las que pasamos a repasar en este trabajo, e incorporamos en anexo final. Es indudable que otras lecciones que aquí no figuran recogen también en algún momento ciertas referencias históricas a las universidades, a veces significativas, pero al no resultar centrales en el conjunto del texto hemos desestimado esas referencias para esta ocasión. Tampoco hemos incluido aquí aquellas lecciones orientadas a la historia de disciplinas académicas concretas, o a las formas didácticas de enseñar, sino que hemos optado por el perfil institucional, incluyendo aquí además de la universidad en su conjunto a las facultades u organismos similares.

La historia de la universidad española en la Fiesta de la Ciencia (1939-2019)

¿Qué información nos arroja el conjunto de las 54 unidades de análisis seleccionadas, publicadas entre 1939 y 2019, para contribuir a enriquecer la historia de las universidades en España?

El reparto en cuanto a las fechas de celebración y publicación de lecciones inaugurales es muy desigual, según universidades. Así, entre 1939 y 1980 solamente escuchan lecciones de historia universitaria las universidades clásicas de Salamanca, Zaragoza, Barcelona, Murcia, Valencia, Sevilla, Valladolid, Oviedo, Universidad Complutense de Madrid, La Laguna, Granada, y en 1979 también las de León y Baleares. Será a partir de 1981 y hasta 2019 cuando, además de las mencionadas, vayan incorporándose universidades de creación más reciente a la presentación en el escenario del respectivo paraninfo del tema de historia de la universidad. Nos referimos a la Universidad Autónoma de Barcelona, Universidad Pública de Navarra, Lérida, Pompeu Fabra, Universidad Politécnica de Cataluña, Universidad Autónoma de Madrid, Coruña, Huelva, Vic, y Extremadura. A estas hay que añadir las privadas confesionales de Comillas, Deusto y Universidad Pontificia de Salamanca, que también aportan lecciones inaugurales sobre algún aspecto de historia de la universidad, generalmente la propia.

De ellas podemos entresacar las universidades más prolíficas en lo que se refiere a discursos inaugurales pronunciados de tema de historia universitaria, que son: Salamanca (6 lecciones), Barcelona (6), Zaragoza (4), Valencia (3), Universidad Autónoma de Barcelona (3), Murcia (2), Universidad Complutense de Madrid (2), Sevilla (2), Valladolid (2), Oviedo (2), Baleares (2), León (2), y con solamente una lección las de La Laguna, Granada, Universidad Pública de Navarra, Universidad Politécnica de Cataluña, Coruña, Huel- 
va, Lleida, Pompeu Fabra, Universidad Autónoma de Madrid, Extremadura, y Vic. A este elenco hemos de añadir las universidades privadas confesionales de Deusto (2), Universidad Pontificia de Salamanca (2) y Comillas (2).

Parece obvio que son las universidades públicas clásicas, algunas de origen medieval, otras nacidas en el periodo renacentista, o a comienzos del siglo XX, las que prestan más interés hacia la historia de la universidad, la propia o la general de España, al menos tal como se desprende de las aportaciones respectivas desde la Fiesta de la Ciencia. Del conjunto de las 54 lecciones estudiadas observamos que 38 de ellas proceden de las universidades clásicas, y solamente 16 a universidades nacidas después de 1969.

Si nos referimos a la lengua en que están escritas y fueron pronunciadas estas lecciones diremos que 13 lo fueron en catalán (Barcelona, Lleida, Baleares, Universidad Autónoma de Barcelona, Universidad Politécnica de Cataluña, Pompeu Fabra y Vic), una lección lo fue en gallego (Coruña), y en el resto de ellas (40) lo hicieron sus autores en castellano.

Hay que expresar que todas excepto una, en la Universidad de Valladolid, expuesta por Fuente Pérez en $2012^{15}$, fueron lecciones pronunciadas por varones. Mientras sabemos que en el conjunto de las 2.165 lecciones analizadas desde nuestra base de datos el 10,3\% fueron obra de mujeres, en este tema de la historia universitaria en lección inaugural la presencia femenina de autoría directa se reduce al 0,54\%.

Podemos entrar a considerar otros aspectos internos de estas lecciones inaugurales, como es, por ejemplo, la temática concreta que se aborda en ellas. Tres de ellas se centran en el análisis de discursos precedentes ya pronunciados en las universidades de Zaragoza por Canellas López en $1969^{16}$ y Salamanca por Lamberto de Echeverría en $1977^{17}$, o bien en las lecciones expuestas y publicadas en las universidades del conjunto de España, como expone en 2016 desde la Universidad de Salamanca José María Hernández Díaz ${ }^{18}$.

${ }^{15}$ Fuente Pérez, María Jesús. El monte Helicón, el Studium Generale de Palencia y los inicios de la universidad. Lección inaugural del curso académico 2012-13 en la Universidad de Valladolid. Valladolid: Universidad de Valladolid, 2012, pp. 63.

${ }^{16}$ Canellas López, Ángel. Paraninfos, 1844-1945. Lección inaugural del curso académico 1969-70 en la Universidad de Zaragoza. Zaragoza: Universidad de Zaragoza, 1969, pp. 87.

${ }^{17}$ Echeverría y Martínez de Marigorta, Lamberto de. De oratoria universitaria salmantina. Oración pronunciada en la Universidad de Salamanca en la solemne apertura del curso académico 1977-78. Salamanca: Universidad de Salamanca, 1977, pp. 100.

${ }^{18}$ Hernández Díaz, José María. La Paideia universitaria en la Fiesta de la Ciencia. Lección inaugural del curso académico 2016-17 en la Universidad de Salamanca. Salamanca: Universidad de Salamanca, 2016, pp. 189. 
Diferentes lecciones inaugurales trasladan vivencias e historias vividas en su propia universidad por los autores, como sucede con la de Valencia en 1961 con Calvo Alfageme ${ }^{19}$, en la Complutense de Madrid en 1985 con Durán Sacristán ${ }^{20}$, en la de Salamanca en 1986 con Manuel Fernández Álvarez $^{21}$, en la Universidad Autónoma de Barcelona en 2012 con Ramón Pascual de Sans ${ }^{22}$, en la de Barcelona en 1959 con José María Pi i Suñer ${ }^{23}$, y antes también en la universidad de Barcelona en 1942 con Enrique Soler y Batlle ${ }^{24}$. Son testimonios de indudable nostalgia, pero también de memoria vivida en su respectiva universidad por los oradores de turno, y en consecuencia una preciosa fuente documental para los investigadores.

En otras ocasiones la lección inaugural es la oportunidad especial para ensalzar determinadas figuras históricas de la propia universidad, que fueron muy distinguidas y especiales en su gestión. Ese es el caso del hermano Gárate en la Universidad de Deusto ensalzado en 1985 por Iturrioz ${ }^{25}$, y de forma destacada en la Universidad de Barcelona del psicólogo y rector Caparrós, del filólogo Joan Corominas y del escritor y poeta Carles Ribas en 2001, 2005 y 2009, respectivamente en la Universidad de Barcelona, a cargo de Siguán, Solá y Cortassa, y Miralles i Solá ${ }^{26}$.

${ }^{19}$ Calvo Alfageme, Álvaro. Estampa universitaria. Lección inaugural del curso 1961-62 en la Universidad de Valencia. Valencia: Universidad de Valencia, 1961, pp. 31.

${ }^{20}$ Durán Sacristán, Hipólito. Vivencias universitarias. Discurso correspondiente a la solemne apertura del curso académico 1985-86 en la Universidad Complutense de Madrid. Madrid: Universidad Complutense, 1985, pp. 76.

${ }^{21}$ Fernández Álvarez, Manuel. Universidad y sociedad. Entre la historia y el recuerdo. Discurso leído en la Universidad de Salamanca en la apertura del curso 1986-87. Salamanca: Universidad de Salamanca, 1986, pp. 66.

${ }^{22}$ Pascual de Sans, Ramón. Els meus temps a la Universitat Autónoma de Barcelona. Lliçó en la inauguració del curs acadèmic 2012-13 en la Universitat Autónoma de Barcelona. Bellaterra: Universitat Autónoma de Barcelona, 2012, pp. 22.

${ }^{23} \mathrm{Pi}$ i Suñer, José María. Mi vieja Facultad de Derecho. Discurso inaugural del año académico 1959-60 en la Universidad de Barcelona. Barcelona: Universidad de Barcelona, 1959, pp. 88.

${ }^{24}$ Soler y Batlle, Enrique. Algunos recuerdos, no muy antiguos, de la Universidad de Barcelona. Discurso inaugural del año académico de 1942-43 en la Universidad de Barcelona. Barcelona: Universidad de Barcelona, 1942, pp. 24.

${ }^{25}$ Iturrioz, Jesús. La Universidad de Deusto, santuario del hermano Gárate. Lección inaugural del curso académico 1985-86 en la Universidad de Deusto. Deusto: Universidad de Deusto, 1985, pp. 40.

${ }^{26}$ Siguán, Miquel. El Rector Caparrós i el futur de la Universitat de Barcelona. Lliçó inaugural del curs 2001-02 en la Universitat de Barcelona. Barcelona: Universitat de Barcelona, 2001, pp.38.; Solá Cortassa, Joan. Joan Corominas com a example. Lliçó inaugural del curs 2005-06 en la Universitat de Barcelona. Barcelona: Universitat de Barcelona, 2005, pp. 38.; Miralles i Sola, 
A veces la temática histórica de la universidad que se analiza se refiere a la universidad del pasado más lejano o distante, como el periodo medieval, el Renacimiento, la Ilustración, incluso el siglo XIX y hasta la guerra civil. Así sucede en las universidades de Salamanca en 1949 con Beneyto Pérez, en 1953 con Madruga Jiménez y en 1957 con Espinosa ${ }^{27}$; en la de Valencia en 1999 con Batllori i Munné, y en 1991 con Santiago Sebastián ${ }^{28}$; en la Universidad de Madrid en 1954 con Carlos Luis de Cuenca ${ }^{29}$; en la Universidad de León en 1982 con Alfonso Prieto Prieto ${ }^{30}$; en la Universidad de Granada en 1989 con Estanislao Olivares ${ }^{31}$; en la Universidad de Lérida en 1992 con Salvador Claramunt Rodríguez ${ }^{32}$; en la Universidad Pública de Navarra en 1996 con la intervención de Juan Carrasco Pérez ${ }^{33}$; y en la de Valladolid en 2012, con la lección ya citada de María Jesús Fuente Pérez,. El resto de lecciones inaugurales puede reconocerse como de mayor proximidad histórica, al situarse en el periodo comprendido entre el inicio de la política universitaria de los liberales a mediados del siglo XIX y la historia próxima al presente.

Carles. Carles Ribas i la universitat. Lliçó inaugural del curs académic 2009-10 en la Universitat de Barcelona. Barcelona: Universitat de Barcelona, 2009, pp. 47.

${ }^{27}$ Beneyto Pérez, Juan. La escuela iluminista salmantina. Discurso leído en la Universidad de Salamanca en la solemne apertura del curso académico 1949-50. Salamanca: Universidad de Salamanca, 1949, pp. 45.; Madruga Jiménez, Esteban. Crónica del Colegio Mayor Arzobispo Fonseca de Salamanca. Discurso leído en las Universidad de Salamanca en la apertura del curso académico de 1953-54. Salamanca: Universidad de Salamanca, 1953, pp. 76.; Espinosa, Ricardo. El Patio de Escuelas. Historia de su construcción. Discurso de apertura del curso académico 1957-58 en la Universidad de Salamanca. Salamanca: Universidad de Salamanca, 1957

${ }^{28}$ Batllori i Munné, Miquel. La Universidad de Valencia en el ámbito cultural de la Corona de Aragón. Lección magistral leída en el solemne acto de apertura del curso 1999-2000 en la Universitat de Valencia. Valencia: Universitat de Valencia, 1999, pp. 27.; Sebastián López, Santiago. La universidad renacentista como palacio de la virtud y del vicio. Discurso leído en la solemne apertura del curso 1991-92 en la Universidad de Valencia. Valencia: Universidad de Valencia, 1991, pp 55.

${ }^{29}$ Luis de Cuenca, Carlos. Sobre el renacimiento español y la universidad hispánica. Discurso pronunciado en la Universidad de Madrid en la apertura del curso 1954 a 1955. Madrid: Artes Gráficas, 1954, pp. 75.

${ }^{30}$ Prieto Prieto, Alfonso. Algunas consideraciones sobre el 'Alma Mater'. Discurso inaugural del curso académico 1982-83 en la Universidad de León. León: Universidad de León, 1982, pp. 127.

${ }^{31}$ Olivares, Estanislao. La docencia de filosofía y teología en el Colegio de San Pablo de Granada (1558-1767). Discurso leído en la solemne apertura del curso académico 1989-90 en la Universidad de Granada. Granada: Universidad de Granada, 1989, pp. 39.

${ }^{32}$ Claramunt Rodríguez, Salvador. La primera universitat de Lleida. Inauguració del Curs Académic 1992-1993. Lliçó magistral a carrec.... Lleida: Universitat de Lleida, 1993, pp. 30.

${ }^{33}$ Carrasco Pérez, Juan. La universidad, una institución de la Edad Media. Lección inaugural en el solemne acto de apertura del curso académico 1996-97 en la Universidad Pública de Navarra. Pamplona: Universidad Pública de Navarra, 1996, pp. 59. 
Un grupo reducido de oradores desde el paraninfo lo hace en pleno huracán posbélico desde 1939, adoptando una posición comprometida, decidida y legitimadora de los vencedores en la contienda, como es el caso de Calamita Álvarez en 1939 en la Universidad de Zaragoza ${ }^{34}$ y en la de Murcia con Montero Díaz ${ }^{35}$, en esa misma fecha de 1939. 0 bien muchos años más tarde se interesa por una revisión actualizada de la posición adoptada por las universidades en aquel conflicto fratricida, como se hace en 2009 desde la Universidad de Huelva a cargo de Juan Carlos Ferré Olivé ${ }^{36}$.

La mayor parte de las lecciones seleccionadas hace un repaso institucional de la universidad que analizan, pero algunas se centran en el estudio de alguna Facultad en particular. Así sucede con la Facultad de Derecho de la Universidad de Barcelona en 1959 con la citada conferencia de Pí Suñer; con el Colegio de Cirugía de Cádiz de la Universidad de Sevilla en 1969, a cargo de Antonio López Rodríguez ${ }^{37}$; la Facultad de Medicina de la Universidad de La Laguna en 1972, escrita por de Víctor Smith-Agreda ${ }^{38}$; la de Veterinaria en la Universidad de Zaragoza en 1998 expuesta por José Antonio Bascuas Asta ${ }^{39}$; el ICAI de la Universidad de Comillas en el año 2008, a cargo de Rafael María Sanz de Diego ${ }^{40}$; con la Facultad de Pedagogía en la Universidad Pontificia de Salamanca en 2010 con la intervención de José Manuel Alfonso Sánchez ${ }^{41}$; o

${ }^{34}$ Calamita Álvarez, Gonzalo. La Universidad de Zaragoza en la Guerra de Liberación. Lección inaugural del curso 1939 a1940 en la Universidad de Zaragoza. Zaragoza: Impr. La Académica, 1939, pp. 35.

${ }^{35}$ Montero Díaz, Santiago. Misión de la universidad. La universidad y los orígenes del nacionalsindicalismo. Discurso de apertura del año académico 1939-40 en la Universidad de Murcia. Murcia: Universidad de Murcia, 1939, pp. 77

${ }^{36}$ Ferré Olivé, Juan Carlos. Universidad y guerra civil. Lección inaugural del curso 2009-10 en la Universidad de Huelva. Huelva: Universidad de Huelva, 2009, pp. 75.

${ }^{37}$ López Rodríguez, Antonio. El Real Colegio de Cirugía de Cádiz y su época. Discurso leído en la solemne apertura del curso académico de 1969-1970 en la Universidad de Sevilla. Sevilla: Universidad de Sevilla, 1969, pp. 59.

${ }^{38}$ Smith-Agreda, Víctor. En torno a la Facultad de Medicina. Lección inaugural del curso 1972-73 en la Universidad de La Laguna. Tenerife: Universidad de la Laguna, 1972, pp. 49.

${ }^{39}$ Bascuas Asta, José Antonio. Pasado reciente y futuro de la Facultad de Veterinaria. Lección inaugural del curso académico 1998-99 en la Universidad de Zaragoza. Zaragoza: Universidad de Zaragoza, 1998, pp. 70

${ }^{40}$ Sanz de Diego, Rafael María. Cimientos sólidos: los primeros años del ICAI (1908-1912) en su centenario. Lección inaugural del curso académico 2008-09 de la Universidad Pontificia Comillas, pronunciada el 1 de octubre de 2008. Madrid: Universidad Pontificia de Comillas, 2008, pp. 56.

${ }^{41}$ Alfonso Sánchez, José Manuel. La Facultad de Pedagogía de la Universidad Pontificia de Salamanca: cincuenta años de historia. Lección inaugural en la solemne apertura del curso académico 2010-11 en la Universidad Pontificia de Salamanca. Salamanca: Universidad Pontificia de Salamanca, 2010, pp. 152. 
por ejemplo con la Facultad de Ciencias de la Universidad de Extremadura, lección inaugural de 2018, obra de Francisco Javier Olivares del Valle ${ }^{42}$.

Algunas lecciones toman como centro temático un Colegio Mayor Universitario, como el Fonseca de Salamanca en la citada lección de Madruga Jiménez de1953, o el de San Pablo de la Universidad de Granada de 1989, según el ya comentado Estanislao Olivares. También una de ellas se permite estudiar en 1974 un centro de práctica clínica médica como es el Hospital Universitario de Sevilla, al tiempo que lo hace del modelo general de docencia práctica para médicos que se está implantando en España en esas fechas. Esta lección fue escrita y pronunciada por Manuel Suárez Perdiguero ${ }^{43}$.

También resulta de gran interés comprobar que la gran mayoría de lecciones aquí estudiadas adoptan como referente de interés central y punto de partida la propia universidad del orador. Así lo confirmamos al ver la elección de la Universidad Pontificia de Salamanca por el ya mencionado José Manuel Alfonso Sánchez en 2010 y Antonio García Madrid ${ }^{44}$, en 2001; la Universidad de Zaragoza en las lecciones referidas de Bascuas Asta (1998), Calamita Álvarez (1939), Canellas López, (1969) y la de Tomeo Lacrué ${ }^{45}$ (1961); la Universidad de Valencia en las analizadas lecciones de Miguel Batllori (1999) y Calvo Alfageme (1961); la Universidad de Salamanca ha sido especialmente visualizada, seguramente por su rica tradición de siglos y vidas académicas, como hemos visto en los discursos inaugurales de Beneyto Pérez(1949), Lamberto de Echeverría (1977), Espinosa (1957); Fernández Álvarez (1986) y Madruga Jiménez (1953); la Universidad de Lérida como comentamos ha sido referenciada por Salvador Claramunt Rodríguez, (1992); la Universidad de Oviedo ${ }^{46}$

${ }^{42}$ Olivares del Valle, Francisco Javier. La Facultad de Ciencias de Extremadura: medio siglo de esperanza y realidades. Lección inaugural del curso académico 2018-19 en la Universidad de Extremadura. Cáceres: Universidad de Extremadura, 2018.

${ }^{43}$ Suárez Perdiguero, Manuel. El Hospital Universitario en la medicina de nuestro tiempo. Discurso de apertura del curso académico 1974-75 en la Universidad de Sevilla. Sevilla: Universidad de Sevilla, 1974, pp. 99.

${ }^{44}$ García Madrid, Antonio. El intento de unificación de las universidades salmantinas en los años 1952 y 1953. Lección inaugural en la solemne apertura del curso académico 2001-02 en la Universidad Pontificia de Salamanca. Salamanca: Universidad Pontificia de Salamanca, 2001, pp. 33

${ }^{45}$ Tomeo Lacrué, Mariano. Biografía científica de la Universidad de Zaragoza. Lección inaugural en la apertura del curso 1961-62 en la Universidad de Zaragoza. Zaragoza: Universidad de Zaragoza, 1961, pp. 115.

${ }^{46}$ Concha Martínez, Ignacio de la. Algunas notas sobre la historia de la universidad. Discurso inaugural del año académico 1978-79 en la Universidad de Oviedo. Oviedo: Universidad de Oviedo, 1978, pp. 18.; Tolívar Alas, Leopoldo. Parlamento y universidad: los senadores de la Universidad de Oviedo. Lección inaugural del curso académico 2019-20 en la Universidad de Oviedo. 
se visibiliza en las intervenciones de Ignacio de la Concha Martínez (1968) y Leopoldo Tolívar Alas, (2019); la Universidad Complutense de Madrid lo hace mediante Durán Sacristán en 1985, ya mencionado; la Universidad de Valladolid tiene sus valedores en María Jesús Fuente Pérez, (2012) y Fidel Mato Vázquez, (1975) ${ }^{47}$; la Universidad de Deusto es difundida, como vimos, por Jesús Iturrioz, en 1985 y más tarde por Adolfo Nicolás ${ }^{48}$, en 2011; la Universidad Pontificia de Comillas en diferentes lecciones inaugurales tiene como valedores al cardenal Jubany ${ }^{49}$ (1991) y como ya mencionamos, a Rafael María Sanz de Diego, (2008); la Universidad de Sevilla nos ofrece a los ya citados profesores Antonio López Rodríguez (1969) y Manuel Suárez Perdiguero (1974); la Universidad de Murcia habla de sí misma en 2008 a través de José Antonio Lozano Teruel ${ }^{50}$; la Universidad de Barcelona muestra varios portavoces particulares (a los ya mencionados Miralles i Solá, (2009), Pí i Suñer (1959), Siguán, (2001), y Solá Cortassa, 2005, hemos de añadir a Enrique Soler y Batlle, (1942) y Ramón Parés i Farrás (1987) ${ }^{51}$; la Universidad de Granada tiene a Estanislo Olivares, (1989), como vimos; la Universidad de Extremadura a Francisco Javier Olivares del Valle (2018), ya revisado; e igualmente la Universidad de La Laguna con Smith-Agreda (1972). Por su parte la Universidad Autónoma de Barcelona ${ }^{52}$, además del ya mencionado Pascual de Sans ( 2012), tiene a Segura i Más, (2008) como relator de la historia de la institución. La Universidad Politécnica de Cataluña se ve realzada en 1995 por Lluis Perman-

Oviedo: Universidad de Oviedo, 2019.

${ }^{47}$ Mato Vázquez, Fidel. La Universidad de Valladolid y la expansión universitaria. Discurso de apertura del curso académico 1975-76 en la Universidad de Valladolid. Valladolid: Universidad de Valladolid, 1975, pp. 43.

${ }^{48}$ Nicolás, Adolfo. A los 125 años de la creación de la Universidad de Deusto. Objetivos de la educación universitaria jesuita. Lección inaugural del curso 2011-12 en la Universidad de Deusto. Bilbao: Universidad de Deusto, 2011.

${ }^{49}$ Jubany i Arnau, Narcís. Comillas, un seminario del Papa en España, 1892-1992. Primer centenario de la universidad. Lección inaugural del curso 1991-92. Madrid, Universidad Pontificia de Comillas, 1991, pp. 21.

${ }^{50}$ Lozano Teruel, José Antonio. Medio siglo de universidad. Lección inaugural del curso académico 2008-09 en la Universidad de Murcia. Murcia: Universidad de Murcia, 2008, pp. 61.

${ }^{51}$ Soler y Batlle, Enrique. Algunos recuerdos, no muy antiguos, de la Universidad de Barcelona. Discurso inaugural del año académico de 1942-43 en la Universidad de Barcelona. Barcelona: Universidad de Barcelona, 1942, pp. 24; Parés i Farrás, Ramón. Meditacions sobre la llibertat amb motiu de la recuperació del lema <libertas > a l'escut de la Universitat de Barcelona. Lliçó de apertura del curs académic 1987-88 en la Universitat de Barcelona.

${ }^{52}$ Segura i Mas, Antoni. La Universitat Autónoma de Barcelona: historia, memoria i compromis. Inauguració del curs acadèmic 2008-09 en la Universitat de Barcelona. Barcelona: Universitat de Barcelona, 2002, pp. 42. 
yer i Lladós ${ }^{53}$. Finalmente, la Universidad de las Islas Baleares también cuenta con su respaldo institucional inicial en este modelo de las lecciones inaugurales, a través de Álvaro Santamaría Arández (1979) ${ }^{54}$.

Otro grupo de lecciones ofrece una proyección más amplia y aplica su estudio al conjunto de la universidad española. Así, lo apreciamos en la Universidad de León en la lección (1979) del prestigioso catedrático de la Facultad de Veterinaria, Justino Burgos, en la que el orador adopta una posición crítica sobre la última etapa de la historia napoleónica de la universidad española y propositiva de mejoras que urgen a la universidad española en el periodo posterior al franquismo, en plena transición política ${ }^{55}$, que en su día fueron novedosas, y en buena medida fueron incorporadas a la Ley de Reforma Universitaria de 1983. En una línea próxima a esta lección se encuentra la que pronuncia también en León en 1982 el profesor Alfonso Prieto Prieto ${ }^{56}$. Alguna lección da un repaso general a la historia de la universidad como institución medieval, como sucede en la Universidad Pública de Navarra, con la de Carrasco Pérez, (1996) ya comentada. Otras lecciones que han sido mencionadas inciden sobre la universidad en la guerra civil y la oratoria, y alguna sobre la universidad hispánica durante el Renacimiento, como en la Universidad de Madrid lo hace Carlos Luis de Cuenca en 1954, y en la de Valencia corre a cargo de Santiago Sebastián López en 1991. El malogrado profesor, víctima de la violencia necia de las armas a causa de la defensa de la libertad plena, el catedrático de historia del derecho Francisco Tomás y Valiente, en su lección pronunciada en 1993 desde la Universidad Autónoma de Madrid esboza un magnífico ensayo sobre la función de la universidad en la construcción del Estado en una perspectiva histórica ${ }^{57}$. Hay quien se atreve, finalmente, desde la Universidad de Vic a una sugerente reflexión de larga duración sobre la Universidad medieval desde su origen en Bolonia hasta el

${ }^{53}$ Permanyer i Lladós, Lluis. 25 anys de la Universitat Politécnica de Catalunya i la historia d'un territori. Lliçó inaugural del curs académic 1995-96 en la Universitat Politécnica de Catalunya. Barcelona: Universitat Politécnica de Catalunya, 1995, pp. 28.

${ }^{54}$ Santamaría Arández, Álvaro. Proceso de institucionalización de la universidad luliana de Mallorca. Lección de apertura del curso académico 1979-80 en la Universidad de las Islas Baleares. Palma de Mallorca: Universidad de las Islas Baleares, 1979, pp. 47.

${ }^{55}$ Burgos González, Justino. Estado actual de la universidad española. Discurso inaugural del año académico 1979-80 en la Universidad de León. León: Universidad de León, 1979, pp. 77.

${ }^{56}$ Prieto Prieto, Alfonso. Algunas consideraciones sobre el 'Alma Mater'. Discurso inaugural del curso académico 1982-83 en la Universidad de León. León: Universidad de León, 1982, pp. 127.

57 Tomás y Valiente, Francisco. Tríptico con prólogo y epílogo. Algunas reflexiones sobre la Universidad, la Historia y el Estado. Lección inaugural del curso académico 1993-94 en la Universidad Autónoma de Madrid. Madrid: Universidad Autónoma de Madrid, 1993, pp. 23. 
plan llamado de Bolonia en 2008, como lo hace en esa misma fecha el médico otorrino Josep Molera Blanch ${ }^{58}$.

En nuestro repaso a los actos de la Fiesta de la Ciencia en diferentes universidades españolas encontramos también un grupo de lecciones inaugurales que podríamos situar expresamente en el sector del nacionalismo reivindicativo, moderado o radical según los casos. Así, se defiende el papel que ha de desempeñar la universidad pública en la construcción de Galicia en su avance económico desde la Universidad de La Coruña, tal como lo expresa en 2008 el economista, empresario y catedrático José María Castellano Ríos $^{59}$. Pero es sobre todo desde varias universidades de Cataluña donde se formula una apuesta nacionalista más decidida, como sucede en la Universidad Autónoma de Barcelona en 1988 a cargo de Josep María Terricabras Nogueras $^{60}$ filósofo y político del partido Esquerra Republicana de Cataluña, y en 1995 de la mano moderada del reconocido médico Josep Laporte i Salas ${ }^{61}$. También es un hecho en la Universidad Pompeu Fabra con la intervención en 1999, de nuevo de parte de Laporte Salas ${ }^{62}$. En términos más moderados también es un hecho en la Universidad de las Islas Baleares con la lección que pronuncia en el año 2000 el profesor historiador, y político liberal, Miquel Durán Pastor ${ }^{63}$.

\section{Significación para la historia de las universidades}

En sí misma, cada una de las lecciones inaugurales pronunciadas en su respectiva universidad construye un eslabón y nuevo peldaño en el edificio de

\footnotetext{
${ }^{58}$ Molera Blanch, Josep. Bolonya 1088-Bolonya 2008. Lliçó inaugural del curs académic 2008-09 en la Universitat de Vic. Vic: Universitat de Vic, 2008, pp. 11.

${ }^{59}$ Castellano Ríos, José María. Reflexions sobre a universidade pública galega. Lección inaugural del curso académico 2008-09 da Universidade da Coruña. A Coruña: Universidade da Coruña, 2008, pp. 87.

${ }^{60}$ Terricabras Nogueras, Josep María. La universitat catalana en el marc europeu. Lliçó d'obertura del curs universitari 1988-89 de la Universitat Autónoma de Barcelona. Barcelona: Universitat Autónoma de Barcelona, 1988, pp. 18.

${ }^{61}$ Laporte i Salas, Josep. La universitat catalana: una reflexió histórica. Lliçó inaugural del curs académic 1995-96 en la Universitat Autónoma de Barcelona. Bellaterra: UAB, 1995, pp. 35.

${ }^{62}$ Laporte i Salas, Josep. La universitat al tomant del segle. Lliçó inaugural del curs académic 1999-2000 en la Universitat Pompeu Fabra. Barcelona: Universitat Pompeu Fabra, 1999, pp. 10.

${ }^{63}$ Durán i Pastor, Miquel. Entre el desconhort y el desencis: el llarg camí de recobrament de la Universitat furtada. Lliçó inaugural del curs 2000-01 en la Universitat de les Illes Balears. Palma: UIB, 2000, pp. 34.
} 
aquella historia particular que se vive en los paraninfos, porque la Fiesta de la Ciencia es una celebración especial, que año tras año va señalando hitos en la historia de la institución universitaria.

Pero, además, para el tema que nos interesa aquí, la historia de la universidad, la aportación que representan estas lecciones inaugurales tiene un valor documental y otro simbólico muy especiales.

Por una parte, muchas de las lecciones inaugurales pronunciadas en los paraninfos realizan aportaciones destacadas de historia universitaria particular y general, según los casos, y entran a formar parte de la historia de esa universidad y a veces de la historia de la universidad española, y en consecuencia de la cultura, la ciencia, la educación de un Estado como es el caso de España. Al respecto, el estudio de la vida universitaria a través del género de la oratoria laica y científica no deja de ser una contribución específica a muchos saberes y ciencias, y en particular para la historia de la universidad, que nunca conviene despreciar.

Por otra parte, no puede desdeñarse el valor eminentemente simbólico de los actos de la Fiesta de la Ciencia, con su protocolo, oropeles, vestimenta, música especial, ceremonias y ritos, paseo académico, presencia de autoridades civiles, militares, eclesiásticas, que hacen que lo que ocurre en el paraninfo de la universidad llegue a alcanzar una proyección externa importante, para los asistentes y para quienes reciben información por medios de prensa escrita, visual y hablada. Al tener la oportunidad de enfatizar algún aspecto de la historia de la universidad, con frecuencia la propia, asistimos a un ejercicio de autoestima colectiva, de autoafirmación en el contexto sociopolítico en que se mueve habitualmente la universidad pública. Estamos, sin duda, ante un acto simbólico, para el interior de la comunidad universitaria, pero también de clara proyección pública y política.

En otro orden de cosas, el reconocimiento a un profesor de la casa, o invitado especial, para pronunciar este discurso inaugural expresa el aplauso a los mejores en sus respectivos campos científicos, pues no es cualquiera quien habla en el paraninfo desde el estrado. Es un reconocimiento moral y sentimental a figuras estelares, pero también a académicos seniors sobre la importancia de una trayectoria científicamente extensa, productiva y honesta, que adopta un perfil ejemplarizante para los más jóvenes y para la sociedad, que ha de quedar incorporada en la historia de la institución universitaria. 


\section{Para finalizar}

Cuando Miguel de Unamuno ${ }^{64}$ dedica en la Universidad de Salamanca el primero de sus discursos inaugurales a los estudiantes en 1900, les animaba al estudio, y su breve pero magistral intervención iba dirigida preferentemente a ellos. De esa forma atendía al sentido que las autoridades políticas y académicas del siglo XIX querían otorgar a ese día tan especial y a esa lección inaugural del curso académico universitario.

A nadie se le oculta que, desde ese sentido prístino de la Fiesta de la Ciencia, buscando casi extenuar a los grupos de alumnos hacia el estudio, y a los profesores a cumplir con su obligación docente, se ha pasado a convertir el acto inaugural del paraninfo en una pequeña feria de vanidades, en la que participan varios actores, y de forma muy diferenciada. Por una parte el rector, equipo de gobierno, séquito académico y de políticos, profesores universitarios, y en nuestros días muy pocos estudiantes presentes en el acto. Paseo ceremonioso $\mathrm{y}$ fotos forman parte inexcusable del protocolo. Por otra hay que mencionar a la figura especial del catedrático elegido ad hoc para pronunciar su lección inaugural, sobre el tema que ha elegido y que ha comentado con su rector con anterioridad. De esa lección queda un documento impreso que pasa al patrimonio colectivo de la universidad, y en muchos casos de los asistentes al acto.

El protocolo es la afirmación del poderoso en todo lugar y cultura del mundo, y la universidad es una institución con poder, que también lo sabe administrar de forma pausada y dosificada. De ahí que el acto inaugural del curso académico en la universidad sea expresión plena de un acto social de celebración in situ, pero también de proyección externa a la sociedad que está en el entorno próximo o el nacional o internacional. Cada acto inaugural, y cada discurso en particular se erige en una expresión de representación simbólica del poder que se cree poseer, exteriorizar y representar.

Al mismo tiempo, y es incuestionable, para uno de los agentes principales de ese protocolo, el orador que pronuncia la lección inaugural, la Fiesta de la Ciencia y su discurso se erigen en un acto poderoso de reconocimiento académico, que quienes tienen la fortuna de poderlo disfrutar nunca van a poder olvidar. En él está en juego el prestigio académico y reconocimiento personal del profesor por la trayectoria seguida en el quehacer universitario, generalmente después de muchos años de dedicación.

${ }^{64}$ Unamuno, Miguel de. Exhortación a la juventud estudiante. Discurso pronunciado en la Universidad de Salamanca en la apertura del cuso 1900 a 1901. Salamanca: Impr. Núñez, 1900, pp. 16. 
Todo esto, es obvio, forma parte de la historia de las universidades, que tiene reservado su espacio en la Fiesta de la Ciencia, y que por ello debe figurar como uno de los elementos de la rica historia de las universidades españolas.

\section{Bibliografía}

Bonilla SAn Martín, Adolfo. La vida corporativa de los estudiantes españoles en sus relaciones con la historia de las universidades. Discurso leído en la Universidad de Madrid en la inauguración del curso 1914 a 1915. Madrid: Impr. Colonial, 1914, pp. 144.

CABEZa De León, SAlvador. La disciplina en la Universidad de Santiago en el siglo XVI-XVIII. Discurso inaugural del curso 1917 a 1918 en la Universidad de Santiago. Santiago de Compostela: Impr. Paredes, 1917, pp. 26.

Domínguez CABREJAS, Rosa. "El discurso pedagógico a través de los paraninfos. Universidad de Zaragoza (1900-1923)", en X COLOQUIO DE HISTORIA DE LA EDUCACION. La Universidad en el siglo XX. España e Iberoamérica. Murcia: Universidad de Murcia, 1998

Fuente, VicENTE DE LA. Sobre el origen de nuestras universidades y su necesidad en todos los tiempos. Discurso que en la solemne inauguración del curso académico de 1856 a 1857 leyó en la Universidad de Salamanca. Salamanca: Imprenta de Telesforo Oliva, 1856.

GoNZÁLEZ DE LA CALLE, URBANO. Estudio de la vida académica y profesional del Brocense en nuestra Escuela. Discurso pronunciado en la Universidad de Salamanca en la inauguración del curso 1912 a 1913. Salamanca, Tip., Núñez, 1912, pp. 192.

HAZAÑAS Y LA RÚA, JOAQUín. La vida escolar universitaria en Sevilla (XVIXVIII). Discurso pronunciado en la Universidad de Sevilla en la inauguración del curso 1907 a 1908. Sevilla: Papelería Sevillana, 1907, pp. 20.

HAZAÑAS Y LA RÚA, JOAQUín. La Universidad de Sevilla. Discurso pronunciado en la Universidad de Sevilla en la inauguración del curso 1922 a 1923. Sevilla, Tip. Eulogio de las Heras, 1922, pp. 33.

HERnÁndez DíAz, José MaRía. La pedagogía en la Fiesta de la Ciencia de la Universidad de Salamanca (1845-1874). REDEX. Revista de Educación de Extremadura. 4,2012, 49-69.

HERNÁNDEZ DíAZ, JosÉ MARÍA. El discurso pedagógico en la Fiesta de la Ciencia en la universidad deseada: España 1900-1936. Historia de la Educación. Revista Interuniversitaria. 34, 2015, 103-138. 
HeRnández Díaz, José María. La Paideia universitaria en la Fiesta de la Ciencia. Salamanca: Universidad de Salamanca, 2016, pp. 189.

Loustau Gómez De Membrillera, José. La Universidad de Murcia y el organismo social. Discurso leído en la inauguración del curso de 1921 a 1922 en la Universidad Literaria de Murcia. Murcia: Imp. José A. Giménez, 1921 pp. 86.

MALDONADo DE GUEVARA, FRANCISCO. Salamanca y su universidad. Discurso pronunciado en la Universidad de Salamanca en la apertura del curso de 1933 a 1934. Salamanca: Impr. Núñez, 1933, pp. 27.

MANOVEl PRIDA; PEDro. Influencia que la Universidad de Salamanca ha tenido en los buenos estudios y en los progresos de las ciencias y las artes. Discurso pronunciado en la Universidad de Salamanca en la apertura del curso 1860 a 1861. Salamanca: Impr. de Diego Vázquez, 1860.

MARTOS DE LA FUENTE, JosÉ. La universidad española hasta el siglo XVIII y sus rasgos característicos. Discurso leído en la Universidad de Granada en la apertura del curso 1928 a 1929. Granada: Tip. López de Guevara, 1928, pp. 44.

Riba García, Carlos. La universidad valentina en los años de la Guerra de la Independencia (1807-1815). Discurso leído en la solemne apertura de los estudios del año académico de 1910 a 1911 de la Universidad de Valencia. Valencia: Universidad de Valencia, 1911, pp. 147.

Romero Díaz, Pedro. Conquistas que el hombre ha hecho en los dominios de la ciencia y beneficios que aquél ha recibido de ésta. Discurso pronunciado en la Universidad de Salamanca en la inauguración del curso 18621863. Salamanca: Impr. de Diego Vázquez, 1862.

RuBIO y BoRRÁs, MANUEL. Aperturas de curso en la Universidad de Barcelona desde 1536 a 1919. Reseña histórica y bibliográfica. Barcelona: Editorial Cultura, 1919.

SERRA RAFols, Elías. Una universidad medieval, el Estudio General de Lérida. Discurso pronunciado en la Universidad de La Laguna en la apertura del curso 1931 a 1932. Madrid: Victoriano Suárez, 1931, pp. 94.

TÉLlez de Meneses SÁnchez, JOSÉ. La Universidad Salmantina y su riqueza bibliográfica. Discurso pronunciado en la Universidad de Salamanca en la apertura del curso académico de 1925 a 1926. Salamanca: Imp. Núñez, 1925.

Torre del Cerro, Antonio de la. Provisión de cátedras en la Universidad de Barcelona de 1559 a 1596. Discurso pronunciado en la Universidad de Barcelona en la inauguración del curso 1926 a 1927. Barcelona: Núñez y Cos, 1926, pp. 26. 
UnAmuno, Miguel DE. Exhortación a la juventud estudiante. Discurso pronunciado en la Universidad de Salamanca en la apertura del cuso 1900 a 1901. Salamanca: Impr. Núñez, 1900, pp. 16.

Anexo. Lecciones inaugurales sobre Historia de la Universidad que se pronuncian en las universidades españolas entre 1939 y 2019

Alfonso Sánchez, José Manuel. La Facultad de Pedagogía de la Universidad Pontificia de Salamanca: cincuenta años de historia. Lección inaugural en la solemne apertura del curso académico 2010-11 en la Universidad Pontificia de Salamanca. Salamanca: Universidad Pontificia de Salamanca, 2010, pp. 152.

Bascuas Asta, José Antonio. Pasado reciente y futuro de la Facultad de Veterinaria. Lección inaugural del curso académico 1998-99 en la Universidad de Zaragoza. Zaragoza: Universidad de Zaragoza, 1998, pp. 70.

Batllori i MunNe, Miquel. La Universidad de Valencia en el ámbito cultural de la Corona de Aragón. Lección magistral leída en el solemne acto de apertura del curso 1999-2000 en la Universitat de Valencia. Valencia: Universitat de Valencia, 1999, pp. 27.

BENEYTo PÉREZ, JuAn. La escuela iluminista salmantina. Discurso leído en la Universidad de Salamanca en la solemne apertura del curso académico 1949-50. Salamanca:Universidad de Salamanca, 1949, pp. 45.

Burgos GonzÁlez, Justino. Estado actual de la universidad española. Discurso inaugural del año académico 1979-80 en la Universidad de León. León: Universidad de León, 1979, pp. 77.

CAlamita ÁlVAREZ, Gonzalo. La Universidad de Zaragoza en la Guerra de Liberación. Lección inaugural del curso 1939 a1940 en la Universidad de Zaragoza. Zaragoza: Impr. La Académica, 1939, pp. 35.

CAlvo Alfageme, Álvaro. Estampa universitaria. Lección inaugural del curso 1961-62 en la Universidad de Valencia. Valencia: Universidad de Valencia, 1961, pp. 31.

CANELlas LóPEZ, Ángel. Paraninfos, 1844-1945. Lección inaugural del curso académico 1969-70 en la Universidad de Zaragoza. Zaragoza: Universidad de Zaragoza, 1969, pp. 87.

Carrasco PÉrez, Juan. La universidad, una institución de la Edad Media. Lección inaugural en el solemne acto de apertura del curso académico 1996-97 en la Universidad Pública de Navarra. Pamplona: Universidad Pública de Navarra, 1996, pp. 59. 
Castellano Ríos, José María. Reflexions sobre a universidade pública galega. Lección inaugural del curso académico 2008-09 da Universidade da Coruña. A Coruña: Universidade da Coruña, 2008, pp. 87.

Claramunt Rodríguez, SAlvador. La primera universitat de Lleida. Inauguració del Curs Académic 1992-1993. Lliçó magistral a carrec.... Lleida: Universitat de Lleida, 1993, pp. 30.

Concha MARTínez, IGNACIO DE LA. Algunas notas sobre la historia de la universidad. Discurso inaugural del año académico 1978-79 en la Universidad de Oviedo. Oviedo: Universidad de Oviedo, 1978, pp. 18.

DURÁN I PASTOR, MiQuel. Entre el desconhort y el desencis: el llarg camí de recobrament de la Universitat furtada. Lliçó inaugural del curs 2000-01 en la Universitat de les Illes Balears. Palma: UIB, 2000, pp. 34.

DURÁN SACRISTÁN, Hipólito. Vivencias universitarias. Discurso correspondiente a la solemne apertura del curso académico 1985-86 en la Universidad Complutense de Madrid. Madrid: Universidad Complutense, 1985, pp. 76.

ECHEVERRÍA Y MARTÍNEZ DE MARIGORTA, LAMBERTo DE. De oratoria universitaria salmantina. Oración pronunciada en la Universidad de Salamanca en la solemne apertura del curso académico 1977-78. Salamanca: Universidad de Salamanca, 1977, pp. 100.

Espinosa, Ricardo. El Patio de Escuelas. Historia de su construcción. Discurso de apertura del curso académico 1957-58 en la Universidad de Salamanca. Salamanca, Universidad de Salamanca, 1957.

FERnÁNDEZ ÁlVAREZ, MANUEL. Universidad y sociedad. Entre la historia y el recuerdo. Discurso leído en la Universidad de Salamanca en la apertura del curso 1986-87. Salamanca: Universidad de Salamanca, 1986, pp. 66.

FERRÉ OLIVÉ, JUAN CARLOS. Universidad y guerra civil. Lección inaugural del curso 2009-10 en la Universidad de Huelva. Huelva: Universidad de Huelva, 2009, pp. 75.

Fuente PÉrez, María Jesús. El monte Helicón, el Studium Generale de Palencia y los inicios de la universidad. Lección inaugural del curso académico 2012-13 en la Universidad de Valladolid. Valladolid: Universidad de Valladolid, 2012, pp. 63.

GARCÍA MADRID, ANTONIO. El intento de unificación de las universidades salmantinas en los años 1952 y 1953. Lección inaugural en la solemne apertura del curso académico 2001-02 en la Universidad Pontificia de Salamanca. Salamanca: Universidad Pontificia de Salamanca, 2001, pp. 33. 
Hernández Díaz, José María. La Paideia universitaria en la Fiesta de la Ciencia. Lección inaugural del curso académico 2016-17 en la Universidad de Salamanca. Salamanca: Universidad de Salamanca, 2016, pp. 189.

ItURRIoz, JEsús. La Universidad de Deusto, santuario del hermano Gárate. Lección inaugural del curso académico 1985-86 en la Universidad de Deusto. Deusto: Universidad de Deusto, 1985, pp. 40.

Jubany i ARnau, NARcís. Comillas, un seminario del Papa en España, 18921992. Primer centenario de la universidad. Lección inaugural del curso 1991-92. Madrid, Universidad Pontificia de Comillas, 1991, pp. 21.

LAPORTE I SALAS, JOSEP. La universitat catalana: una reflexió histórica. Lliçó inaugural del curs académic 1995-96 en la Universitat Autónoma de Barcelona. Bellaterra: UAB, 1995, pp. 35.

LAPORTE I SALAS, JOSEP. La universitat al tomant del segle. Lliçó inaugural del curs académic 1999-2000 en la Universitat Pompeu Fabra. Barcelona: Universitat Pompeu Fabra, 1999, pp. 10.

López Rodríguez, Antonio. El Real Colegio de Cirugía de Cádiz y su época. Discurso leído en la solemne apertura del curso académico de 1969-1970 en la Universidad de Sevilla. Sevilla: Universidad de Sevilla, 1969, pp. 59.

LOZANo TERUEL, José ANTONIO. Medio siglo de universidad. Lección inaugural del curso académico 2008-09 en la Universidad de Murcia. Murcia: Universidad de Murcia, 2008, pp. 61.

LUIS DE CUENCA, CARLOS. Sobre el renacimiento español y la universidad hispánica. Discurso pronunciado en la Universidad de Madrid en la apertura del curso 1954 a 1955. Madrid: Artes Gráficas, 1954, pp. 75.

Madruga Jiménez, Esteban. Crónica del Colegio Mayor Arzobispo Fonseca de Salamanca. Discurso leído en las Universidad de Salamanca en la apertura del curso académico de 1953-54. Salamanca: Universidad de Salamanca, 1953, pp. 76.

Mato VÁzQUez, Fidel. La Universidad de Valladolid y la expansión universitaria. Discurso de apertura del curso académico 1975-76 en la Universidad de Valladolid. Valladolid: Universidad de Valladolid, 1975, pp. 43.

MIRALles I Sola, CARLES. Carles Ribas i la universitat. Lliçó inaugural del curs académic 2009-10 en la Universitat de Barcelona. Barcelona: Universitat de Barcelona, 2009, pp. 47.

Molera Blanch, Joser. Bolonya 1088-Bolonya 2008. Lliçó inaugural del curs académic 2008-09 en la Universitat de Vic. Vic: Universitat de Vic, 2008, pp. 11.

Montero Díaz, SAntiago. Misión de la universidad. La universidad y los orígenes del nacionalsindicalismo. Discurso de apertura del año académico 
1939-40 en la Universidad de Murcia. Murcia: Universidad de Murcia, 1939 , pp. 77.

Nicolás, Adolfo. A los 125 años de la creación de la Universidad de Deusto. Objetivos de la educación universitaria jesuita. Lección inaugural del curso 2011-12 en la Universidad de Deusto. Bilbao: Universidad de Deusto, 2011.

Olivares, Estanislao. La docencia de filosofía y teología en el Colegio de San Pablo de Granada (1558-1767). Discurso leído en la solemne apertura del curso académico 1989-90 en la Universidad de Granada. Granada: Universidad de Granada, 1989, pp. 39.

Olivares del Valle, Francisco Javier. La Facultad de Ciencias de Extremadura: medio siglo de esperanza y realidades. Lección inaugural del curso académico 2018-19 en la Universidad de Extremadura. Cáceres: Universidad de Extremadura, 2018.

PARÉS I FARRÁs, RAMÓN. Meditacions sobre la llibertat amb motiu de la recuperació del lema <libertas> a l'escut de la Universitat de Barcelona. Lliçó de apertura del curs académic 1987-88 en la Universitat de Barcelona. Barcelona: Universitat de Barcelona, 1987, pp. 36.

PASCUAL DE SANS, RAmón. Els meus temps a la Universitat Autónoma de Barcelona. Lliçó en la inauguració del curs acadèmic 2012-13 en la Universitat Autónoma de Barcelona. Bellaterra: Universitat Autónoma de Barcelona, 2012, pp. 22.

Permanyer I Lladós, Lluis. 25 anys de la Universitat Politécnica de Catalunya i la historia d'un territori. Lliçó inaugural del curs académic 1995-96 en la Universitat Politécnica de Catalunya. Barcelona: Universitat Politécnica de Catalunya, 1995, pp. 28.

Pi I Suñer, José María. Mi vieja Facultad de Derecho. Discurso inaugural del año académico 1959-60 en la Universidad de Barcelona. Barcelona: Universidad de Barcelona, 1959, pp. 88.

Prieto Prieto, Alfonso. Algunas consideraciones sobre el 'Alma Mater'. Discurso inaugural del curso académico 1982-83 en la Universidad de León. León: Universidad de León, 1982, pp. 127.

Santamaría Arández, Álvaro. Proceso de institucionalización de la universidad luliana de Mallorca. Lección de apertura del curso académico 197980 en la Universidad de las Islas Baleares. Palma de Mallorca: Universidad de las Islas Baleares, 1979, pp. 47.

SANZ De Diego, Rafael María. Cimientos sólidos: los primeros años del ICAI (1908-1912) en su centenario. Lección inaugural del curso académico 2008-09 de la Universidad Pontificia Comillas, pronunciada el 1 de 
octubre de 2008. Madrid: Universidad Pontificia de Comillas, 2008, pp. 56.

Sebastián López, SANTIAgo. La universidad renacentista como palacio de la virtud y del vicio. Discurso leído en la solemne apertura del curso 199192 en la Universidad de Valencia. Valencia: Universidad de Valencia, 1991 , pp 55.

SEgURA I MAS, ANTONI. La Universitat Autónoma de Barcelona: historia, memoria i compromis. Inauguració del curs acadèmic 2008-09 en la Universitat de Barcelona. Barcelona: Universitat de Barcelona, 2002, pp. 42.

Siguán, Miquel. El Rector Caparrós i el futur de la Universitat de Barcelona. Lliçó inaugural del curs 2001-02 en la Universitat de Barcelona. Barcelona: Universitat de Barcelona, 2001, pp.38.

SMith-Agreda, Víctor. En torno a la Facultad de Medicina. Lección inaugural del curso 1972-73 en la Universidad de La Laguna. Tenerife: Universidad de la Laguna, 1972, pp. 49.

SOlÁ CoRTASSA, JOAN. Joan Corominas com a example. Lliçó inaugural del curs 2005-06 en la Universitat de Barcelona. Barcelona: Universitat de Barcelona, 2005, pp. 38.

SOlER y BATLle, ENRIQUe. Algunos recuerdos, no muy antiguos, de la Universidad de Barcelona. Discurso inaugural del año académico de 1942-43 en la Universidad de Barcelona. Barcelona: Universidad de Barcelona, 1942, pp. 24.

SuÁrez Perdiguero, Manuel. El Hospital Universitario en la medicina de nuestro tiempo. Discurso de apertura del curso académico 1974-75 en la Universidad de Sevilla. Sevilla: Universidad de Sevilla, 1974, pp. 99.

TERRICABRAS NOGUERAS, JOSEP MARÍA. La universitat catalana en el marc europeu. Lliçó d'obertura del curs universitari 1988-89 de la Universitat Autónoma de Barcelona. Barcelona: Universitat Autónoma de Barcelona, 1988, pp. 18

Tolívar Alas, LEOPoldo. Parlamento y universidad: los senadores de la Universidad de Oviedo. Lección inaugural del curso académico 2019-20 en la Universidad de Oviedo. Oviedo: Universidad de Oviedo, 2019.

Tomás y VALIEnTE, Francisco. Tríptico con prólogo y epílogo. Algunas reflexiones sobre la Universidad, la Historia y el Estado. Lección inaugural del curso académico 1993-94 en la Universidad Autónoma de Madrid. Madrid: Universidad Autónoma de Madrid, 1993, pp. 23.

TOMEO LACRUÉ, MARIANo. Biografía científica de la Universidad de Zaragoza. Lección inaugural en la apertura del curso 1961-62 en la Universidad de Zaragoza. Zaragoza: Universidad de Zaragoza, 1961, pp. 115. 